\title{
Bergapten induces metabolic reprogramming in breast cancer cells
}

\author{
MARTA SANTORO ${ }^{1-3}$, CARMELA GUIDO $^{1-3}$, FRANCESCA DE AMICIS $^{1,3}$, DIEGO SISCI $^{1,3}$, ERIKA CIONE $^{3}$, \\ DOLCE VINCENZA $^{3}$, ADA DONÀ ${ }^{1,3}$, MARIA LUISA PANNO $^{3 *}$ and SAVERIA AQUILA ${ }^{1,3^{*}}$ \\ ${ }^{1}$ Centro Sanitario, ${ }^{2}$ Post-Graduate School Clinical Pathology, ${ }^{3}$ Department of Pharmacy and Science of Health \\ and Nutrition, University of Calabria, I-87030 Arcavacata di Rende (CS), Italy
}

Received March 13, 2015; Accepted August 4, 2015

DOI: $10.3892 / o r .2015 .4327$

\begin{abstract}
Alterations in cellular metabolism are among the most consistent hallmarks of cancer. Herein, after a comprehensive metabolic phenotype characterization of MCF7 and ZR75 breast cancer cells, we investigated the activity of bergapten $(\mathrm{Bg})$, a plant-derived compound, against breast cancer. The study of different biochemical pathways involved in cell metabolism revealed that the two cell lines have different bioenergetic phenotypes: MCF7 cells express a glycolytic phenotype only partially oxidative, while ZR75 cells mainly have an oxidative phenotype. In both cell lines, Bg blocked glycolysis and significantly decreased glucose-6-phosphate dehydrogenase (G6PDH) activity promoting glucose accumulation; modulated bioenergetic requirements altering the expression of oxidative phosphorylation (OXPHOS) complexes and ATP production; and induced a lipid-lowering effect since an increased lipase activity concomitantly to a reduction in triglyceride levels was observed. Quantitative data of different metabolites and enzymatic activities were presented. Treatment with $\mathrm{Bg}$ resulted in an alteration in different metabolic pathways inducing death in the cells. We report a novel action of the natural product $\mathrm{Bg}$ on breast cancer, since it induced metabolic reprogramming by disrupting the interconnected network of different metabolic mechanisms. Bg can be used in combination with other forms of targeted chemotherapy to improve cancer treatment outcomes.
\end{abstract}

Correspondence to: Professor Saveria Aquila, Department of Pharmacy and Science of Health and Nutrition, University of Calabria, I-87036 Arcavacata di Rende (CS), Italy

E-mail: saveria.aquila@libero.it; mamissina@yahoo.it

"Joint senior authorship

Abbreviations: $\mathrm{Bg}$, bergapten; $\mathrm{LDH}$, lactate dehydrogenase; G6PDH, glucose-6-phosphate dehydrogenase; OXPHOS, oxidative phosphorylation

Key words: cancer metabolism, lactate dehydrogenase, lipase activity, glucose-6-phosphate dehydrogenase activity, OXPHOS, methoxypsoralen

\section{Introduction}

Recently, energy metabolism has been considered an attractive target for antitumoral therapies, since metabolic changes are a common feature of cancerous tissues. Multiple molecular mechanisms, both intrinsic and extrinsic, converge to alter cellular metabolism. The best characterized metabolic phenotype observed in tumor cells is the Warburg effect, which involves ATP generation through glycolysis instead of oxidative phosphorylation (OXPHOS), even under normal oxygen concentrations (1). However, metabolic adaptation in tumors extends beyond the Warburg effect. In fact, the classical theory on the metabolism of cancer cells (increased glycolytic activity and downregulation of OXPHOS) is still under investigation since different studies have shown that cancer cells can live in a wide spectrum of states ranging from the predominance of the glycolytic phenotype to a phosphorylative phenotype (2). Complete catabolism of pyruvate to $\mathrm{CO}_{2}$ may be counterproductive in a dividing cell as it may limit the availability of precursors necessary for cell replication, e.g., sugars for nucleotide biosynthesis.

In normal cells, glucose is catabolized to pyruvate, which can be later converted to acetyl-CoA to fuel the tricarboxylic acid cycle. On the other hand, the manner by which tumor cells process pyruvate, the end product of glycolysis, is also different from normal cells, since pyruvate-to-lactate is a preferred reaction in tumor cells (3). Therefore, in cancer cells, the glycolytic flux may be blocked at pyruvate production by pyruvate kinase. In this case, pyruvate is no longer oxidized in mitochondria and is metabolized by cytosolic lactate dehydrogenase. Neverthess, a portion of pyruvate is imported into mitochondria and it still might be metabolized through a mitochondrial oxidative phosphorylative pathway (OXPHOS), creating the partial OXPHOS phenotype.

Differences in OXPHOS status originate from variability of metabolic reprogramming among cancer cells and from the relative contributions of different oncogenes, tumor environment, and mitochondrial function. Disruption of cancer cell metabolism represents an elegant approach to induce tumoral cell death; indeed cell death and metabolic alteration are closely related. The strategy to target tumor metabolism requires an integrated vision of this process since it is not a single metabolic step that is altered; but the entire energetic metabolism working in a pattern is profoundly affected with respect to normal cells. In addition, the metabolic alterations 
and adaptations of cancer cells create a particular phenotype essential for tumor cell growth and survival that is particular for each type of cancer. Therefore, it may help to understand how, step by step, the metabolic pathways are arranged in comparison with normal metabolism to characterize a cancer metabolic phenotype.

Several drugs could be referred to as mitocans, metabocans, or aberrocans and many are being developed at present (4). Recently, several studies have documented the ability of chemopreventive phytochemicals to increase the sensitivity of tumoral cells to anticancer drugs $(5,6)$. Bergapten (5-methoxypsoralen, $\mathrm{Bg}$ ) is a psoralen or taxol (also known as furocoumarins) found in bergamot essential oil and in other citrus essential oils. It belongs to the flavonoid class, which has been found to exhibit a variety of biological activities, usually associated with low toxicity. Flavonoids have attracted considerable interest because of their diverse pharmacological properties. Bg activity against different types of tumor including prostate, ovarian, lung cancer and breast cancer has been observed (7).

It is generally believed that the antitumor effect of $\mathrm{Bg}$ is due to its interference with the normal function of microtubuleinducing arrest of the cell cycle (7). However, studies have shown that taxol-induced cell death is via multiple signaling pathways (8-11). For example, taxol-induced apoptosis in breast cancer cells requires downregulation of $\mathrm{I} \kappa \mathrm{B} \alpha$, which in turn activates NF- $\kappa \mathrm{B}(10)$. Activation of MAPK pathways by taxol in HeLa cells was found to lead to a time-dependent increase in poly(ADP-ribose) polymerase cleavage and apoptosis (11). A study of differential gene expression in mouse cells treated with taxol identified a number of genes that modulate apoptosis (9). We previously demonstrated that in breast cancer cells, bergapten $(\mathrm{Bg})$ treatment affects the PI3K/ AKT survival pathway, inducing apoptosis and lowering cell proliferation (12). In addition, we found that $\mathrm{Bg}$ enhanced $\mathrm{p} 53$ gene expression inducing apoptosis in breast cancer cells (13).

Herein, we report a new anticancer effect of $\mathrm{Bg}$ on human breast cancer, since it was able to interfere in cancer reprogrammed metabolism. We characterized the basal metabolic profile of MCF7 and ZR75 cells and evaluated the effect of Bg on different metabolic pathways. Moreover, we present various quantitative aspects of human breast cancer cell metabolism which are limited by the lack of available data at the level of metabolite amounts and enzymatic activities. The quantification can yield multiple insights into the organization of the cancer metabolic network.

\section{Materials and methods}

Materials. Methoxypsoralen or $\mathrm{Bg}$, aprotinin, leupeptin, phenylmethylsulfonyl fluoride (PMFS), sodium orthovanadate, Tris- $\mathrm{Cl}, \mathrm{MnCl}_{2}, \mathrm{NADP}^{+}$, isocitrate and malate were obtained from Sigma-Aldrich (Milan, Italy). Antibodies used in this study were from Santa Cruz Biotechnology (Santa Cruz, CA, USA). MitoProfile ${ }^{\circledR}$ Total OXPHOS WB Antibody Cocktail was from Abcam (Milan, Italy). Triglycerides, lipase activity, glucose-6-phosphate dehydrogenase (G6PDH) activity, LDH activity and glucose assay kits were from Inter-Medical (Biogemina Italia Srl, Catania, Italy). Molecular Probes' ATP Determination kit (A22066) was from Invitrogen (Milan,
Italy). $\mathrm{Bg}$ was dissolved in ethanol ( $0.02 \%$ final concentration) which was used as a solvent control and did not induce any positive results in all in vitro assays (data not shown).

Cell culture. Human breast cancer MCF7 and ZR75-1 (ZR75) cells (American Type Culture Collection, ATCC) were stored according to supplier's instructions. Every 4 months, cells were authenticated by single tandem repeat analysis at our Sequencing Core; morphology, doubling times, estrogen sensitivity, and mycoplasma negativity were tested (MycoAlert; Lonza). Both cell lines were maintained in DMEM/F-12 medium containing $5 \%$ fetal calf serum (FCS), $1 \%$ L-glutamine, $1 \%$ Eagle's non-essential amino acids and $1 \mathrm{mg} / \mathrm{ml}$ penicillin/ streptomycin in a $5 \% \mathrm{CO}_{2}$ humidified atmosphere. Cells cultured in phenol red and serum-free medium for $24 \mathrm{~h}$, were treated with $\mathrm{Bg}$ in medium containing $5 \%$ charcoal-treated FCS. The Bg concentrations were chosen on the basis of our previous studies $(12,13)$. In addition, we found that Bg induces apoptosis at $24 \mathrm{~h}$, therefore we hypothesized that a metabolic disruption may occur in a shorter time period, thus we treated the cells for 6 and $16 \mathrm{~h}$ (12).

Western blotting. Total protein extracts were obtained as previously described (14). Proteins were resolved on a $10 \%$ sodium dodecyl sulfate-polyacrylamide gel, transferred to a nitrocellulose membrane, and probed overnight at $4^{\circ} \mathrm{C}$ with the indicated antibodies. $\beta$-actin was used as loading control.

Glucose assay. Glucose oxidase catalyzes the oxidation of glucose to gluconic acid. The formed hydrogen peroxide is detected by a chromogenic oxygen acceptor, phenol, 4-aminophenazone in the presence of peroxidase. The intensity of the color formed is proportional to the glucose concentration in the sample (14). Data are presented as $\mathrm{nM} / \mathrm{mg}$ protein.

LDH assay. LDH catalyzes the interconversion of pyruvate and lactate with concomitant interconversion of NADH and $\mathrm{NAD}^{+}$. After the treatments, $\mathrm{LDH}$ assay was performed on cell lysates as previously described (13). Data are presented as absorbance change at $340 \mathrm{~nm}$.

Triglyceride assay. Triglycerides were measured in duplicate by a GPO-POD enzymatic colorimetric method according to the manufacturer's instructions in cell lysates and as previously described (16). Data are presented as $\mathrm{nM} / \mathrm{mg}$ protein.

Lipase activity assay. Lipase activity was evaluated by the method of Panteghini et al (17) based on the use of 1,2-o-dilauryl-rac-glycero-3-glutaric acid-(6'-methylresorufin) ester as substrate, as previously described (18). Data are presented as $\mathrm{nM} / \mathrm{min} / \mathrm{mg}$ protein.

Assay of the G6PDH activity. The conversion of $\mathrm{NADP}^{+}$to NADPH, catalyzed by G6PDH, was measured by the increase in absorbance at $340 \mathrm{~nm}$ as previously described $(16,18)$. Data are presented as $\mathrm{nM} / \mathrm{min} / \mathrm{mg}$ protein.

ATP assay. A bioluminescence assay for quantitative determination of ATP with recombinant firefly luciferase and its substrate D-luciferin (light emission at $560 \mathrm{~nm}$ at $\mathrm{pH} 7.8$ ), was 
A

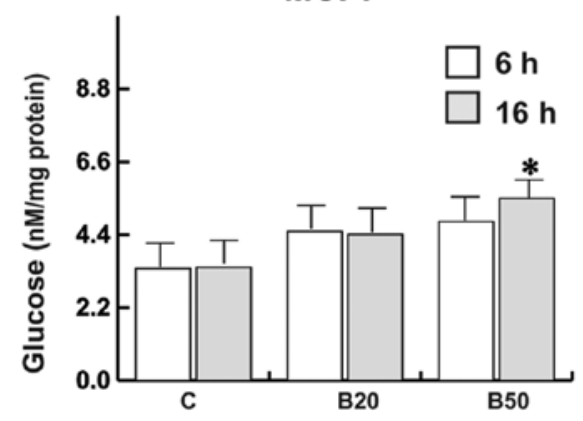

B

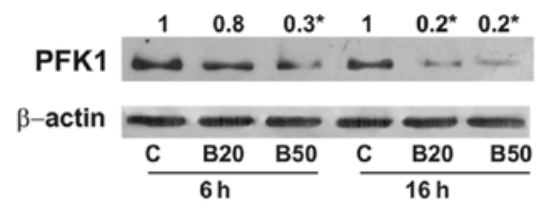

C

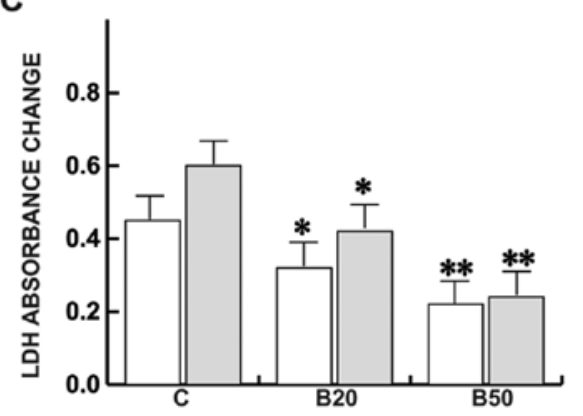

ZR75
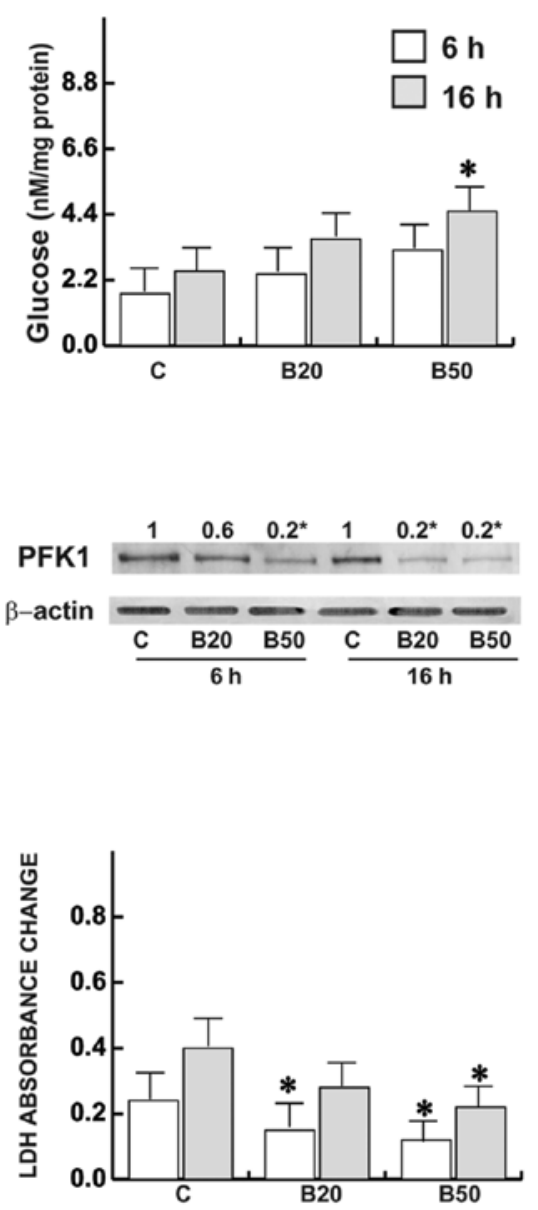

Figure 1. Bg disrupts glycolysis in breast cancer cells. (A) Glucose content was assessed as reported in Materials and methods and in both cell lines in the absence (C) or with increasing Bg concentrations (B20 $\mu \mathrm{M}$ and B50 $\mu \mathrm{M})$ at the indicated times. Columns represent the mean \pm SEM of 20 independent experiments performed in duplicate. "P $<0.05$ vs. control. (B) Western blot assay of PFK1 in both cell lines in the absence (C) or with increasing Bg concentrations $(\mathrm{B} 20 \mu \mathrm{M}$ and $\mathrm{B} 50 \mu \mathrm{M})$ at the indicated times. $\beta$-actin was used as control. The autoradiographs show the results of one representative experiment, and the numbers on the top of the blot, are the mean of three independent experiments in which band intensities were evaluated in terms of optical density arbitrary units and expressed as the fold over each control (C), which was assumed to be $1 .{ }^{*} \mathrm{P}<0.01$ vs. the control (C) at the same time. (C) LDH activity was assessed as reported in Materials and methods. Columns represent the mean $\pm \mathrm{SEM}$ of 20 independent experiments performed in duplicate. ${ }^{*} \mathrm{P}<0.05 ;{ }^{* *} \mathrm{P}<0.02$ vs. control (C) at the same time. Bg, bergapten; LDH, lactate dehydrogenase.

performed as previously described (19). Data are presented as $\mathrm{nM} / \mathrm{mg}$ protein.

Isocitrate dehydrogenase (ICD) activity assay. ICD activity was measured as in Gnoni and Paglialonga (20). The reaction was performed on the cell lysate using a final 1-ml reaction volume containing $0.2 \mathrm{mg}$ protein, $50 \mathrm{mM}$ Tris- $\mathrm{Cl} \mathrm{pH} \mathrm{7.4,}$ $5 \mathrm{mM} \mathrm{MnCl}_{2}, 0.25 \mathrm{mM} \mathrm{NADP}^{+}$and $0.25 \mathrm{mM}$ isocitrate, at $37^{\circ} \mathrm{C}$. Reaction progress was monitored at $340 \mathrm{~nm}$ for $2 \mathrm{~min}$; the NADPH production was calculated using an NADPH extinction coefficient of $6.26103 \mathrm{M}^{-1} \mathrm{~cm}^{-1}$. Data are presented as $\mathrm{nM} / \mathrm{min} / \mathrm{mg}$ protein.

Malic enzyme (ME) activity assay. ME activity was measured in the cell lysates as in Gnoni and Paglialonga (20). The reaction was performed using a final 1-ml reaction volume containing $0.3 \mathrm{mg}$ protein, $50 \mathrm{mM}$ Tris- $\mathrm{HCl} \mathrm{pH} \mathrm{7.4,} 5 \mathrm{mM}$ $\mathrm{MnCl}_{2}, 0.1 \mathrm{mM} \mathrm{NADP}{ }^{+}$and $5 \mathrm{mM}$ malate, at room temperature. Reaction progress was monitored at $340 \mathrm{~nm}$ for $2 \mathrm{~min}$; the NADPH production was calculated using an NADPH extinction coefficient of $6.26103 \mathrm{M}^{-1} \mathrm{~cm}^{-1}$. Data are presented as $\mathrm{nM} / \mathrm{min} / \mathrm{mg}$ protein.

Trypan blue assay. Cells were treated as indicated and the loss of survival was assessed by the trypan blue assay as previously described (14).

Statistical analysis. Each datum point represents the mean \pm SEM of the experimental values as indicated in the figure legends. Data were analyzed by the Student's t-test using the GraphPad Prism 4 software program. $\mathrm{P}<0.05$ was considered to indicate a statistically significant result.

\section{Results}

Bg interferes with the glycolysis in MCF7 and ZR75 cells. To characterize the basal metabolic phenotype of both breast cancer cell lines as well as following Bg treatment, we first 
A

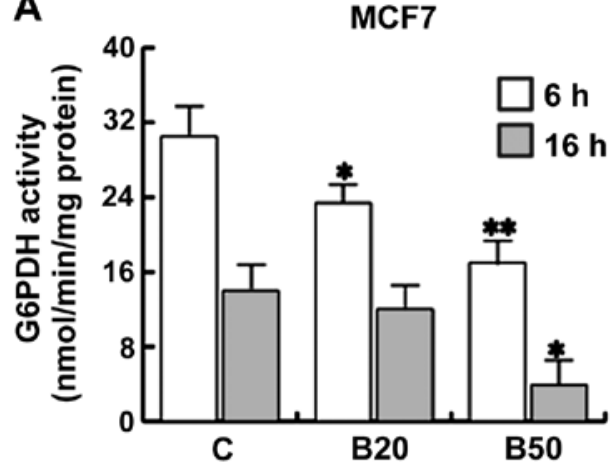

B

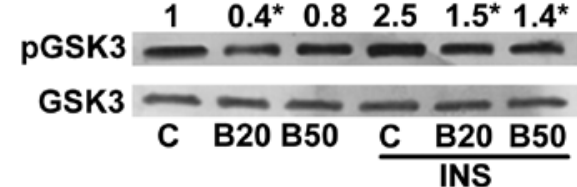

ZR75
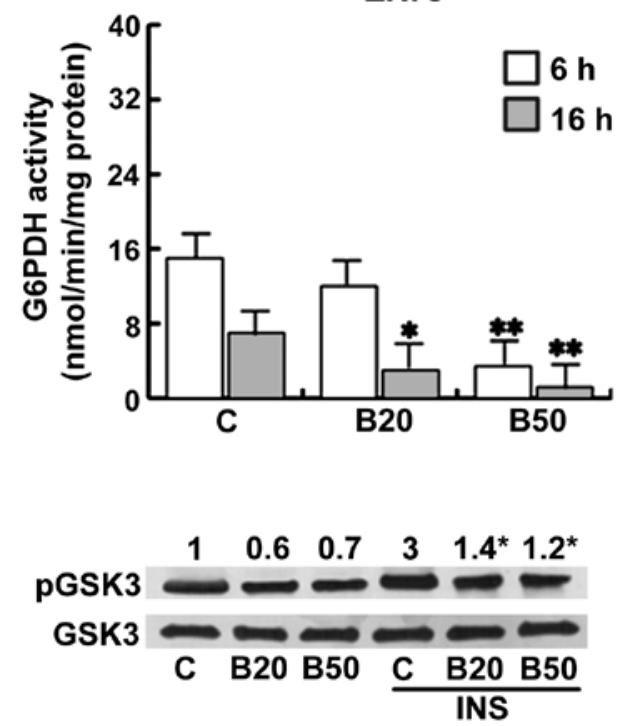

Figure 2. Bg modifies glucose utilization in breast cancer cells. (A) G6PDH activity was performed as reported in Materials and methods and in both cell lines in the absence $(\mathrm{C})$ or with increasing Bg concentrations (B20 $\mu \mathrm{M}$ and B50 $\mu \mathrm{M})$ at the indicated times. Columns represent the mean \pm SEM of 20 independent experiments performed in duplicate. ${ }^{*} \mathrm{P}<0.05,{ }^{* *} \mathrm{P}<0.01$ vs. the control (C) at the same time. (B) Western blot analysis of pGSK3S9 and GSK3 in MCF7 and ZR75 cells cultured as indicated. INS, insulin $100 \mathrm{nM}$. The autoradiographs show the results of one representative experiment, and the numbers on the top of the blot are the mean of 3 independent experiments in which band intensities were evaluated in terms of optical density arbitrary units and expressed as the fold over control (C), which was assumed to be 1 . ${ }^{*} \mathrm{P}<0.05$ vs. the control (C) at the same time. Bg, bergapten.

determined the glucose content and analyzed various key enzymes involved in the carbohydrate metabolism: 6-phosphofructo-1-kinase (PFK1) expression and the LDH activity. Bg produced a slight glucose accumulation although it was significant following treatment with $\mathrm{Bg}$ at $50 \mu \mathrm{M}$ at $16 \mathrm{~h}$ in both cell types (Fig. 1A). The cells had decreased glucose utilization after $16 \mathrm{~h}$, and this commensurated with inhibition of cell viability as reported in previous studies $(12,13)$; while a drastic reduction in the glycolytic enzyme PFK1 was observed following treatment with Bg at 20 and $50 \mu \mathrm{M}$ (Fig. 1B). In the same experimental conditions, lactate production significantly decreased (Fig. 1C), both at 6 and 16 h. Noteworthy, ZR75 cells possessed lower basal LDH activity as well as glucose content with respect to the MCF7 cells.

Effects of Bg on biosynthetic contributions in MCF7 and ZR75 cells. Another manner of glucose utilization is via the pentose phosphate cycle (PPP), through G6PDH activity. Bg treatment induced a significant reduction in the enzymatic activity (Fig. 2A), indicating how the decrease in G6PDH in treated cells contributes to the decrease in glucose utilization. Interestingly, ZR75 cells possessed a lower basal G6PDH activity with respect to the MCF7 cells. Glycogen represents an alternative energy source in tumors expressing active glycogen synthase (20). Phosphorylation of glycogen synthase by GSK3 phosphorylated on Ser9 inhibits glycogen synthesis. In the present study, Bg was able to reduce this GSK3 phosphorylation, implying a blockade on the glycogen synthase activity. GSK3 has been initially identified as a key regulator of insulin-dependent glycogen synthesis and we found that $\mathrm{Bg}$ antagonized the upregulatory effect elicited by insulin on this enzyme (Fig. 2B).

Therefore, we decided to further investigate the effect of $\mathrm{Bg}$ on the bioenergetic balance of breast cancer cells. Two enzymes involved in the production of NADPH other than PPP, ME and ICDH, were studied. In MCF7 cells, (Fig. 3A and B), treatment of $\mathrm{Bg}$ at $20 \mu \mathrm{M}$ was able to induce the activities of both enzymes, while treatment with $\mathrm{Bg}$ at $50 \mu \mathrm{M}$ reduced ME activity although not significantly; the extension of treatment with $\mathrm{Bg}$ at $50 \mu \mathrm{M}$ for up to $16 \mathrm{~h}$ induced a significant reduction in ICDH activity. A similar pattern of response was obtained in ZR75 cells upon treatment of $\mathrm{Bg}$ at $20 \mu \mathrm{M}$; however, following treatment with Bg at $50 \mu \mathrm{M}$, the enzymatic activities were close to that of the controls. Notably, ZR75 cells possessed lower basal enzymatic activities with respect to the MCF7 cells.

Bg modulates bioenergetic requirements in MCF7 and ZR75 cells. Successively, we focused our investigation on OXPHOS, by analyzing the mitochondrial OXPHOS components, the ATP content and the AMP-activated protein kinase (AMPK) expression.

Some of the OXPHOS components were not affected by Bg treatment in the MCF7 cells, except for Complex (C) IV that resulted in its upregulation, while CI was reduced particularly at $16 \mathrm{~h}$ (Fig. 4A and B). These data, as expected, resulted in a decrease in ATP production (Fig. 4C). Concerning ZR75 cells, the OXPHOS protein pattern at basal conditions and under $\mathrm{Bg}$ was expressed to a greater extent with respect to the MCF7 cells, although starting from the same protein loading. Even when the CIV component was poorly expressed with respect to the other components in ZR75 cells, it ensured the flow of electrons along the other components, that were overexpressed (including the CII) maintaining a high ATP synthesis, as shown in Fig. 4C. The CIV component of the mitochondrial respiratory chain was expressed to a lesser extent in the ZR75 cells with respect to the MCF7 cells.

AMPK is activated by metabolic stresses that interfere with ATP production (2). In basal conditions the levels of ATP 
A

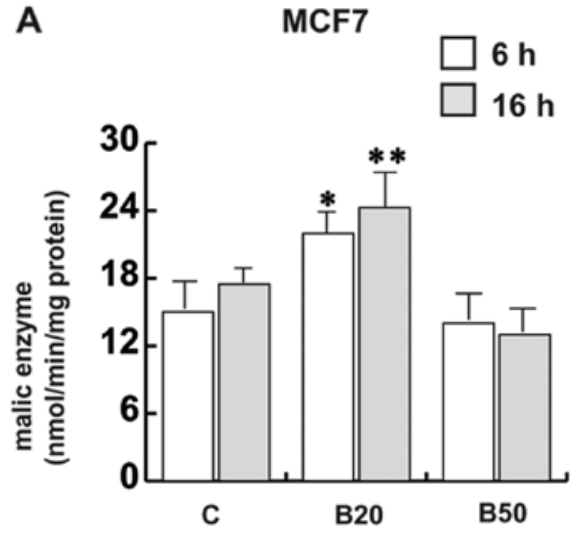

B

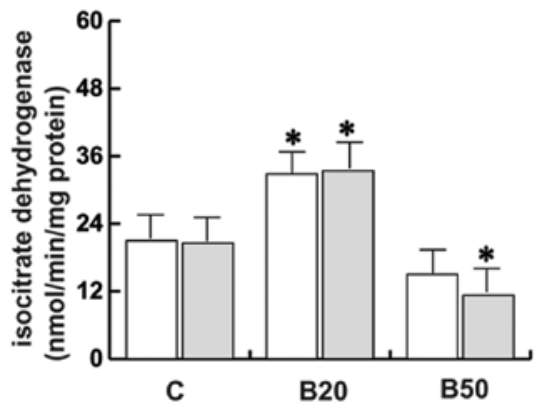

ZR75
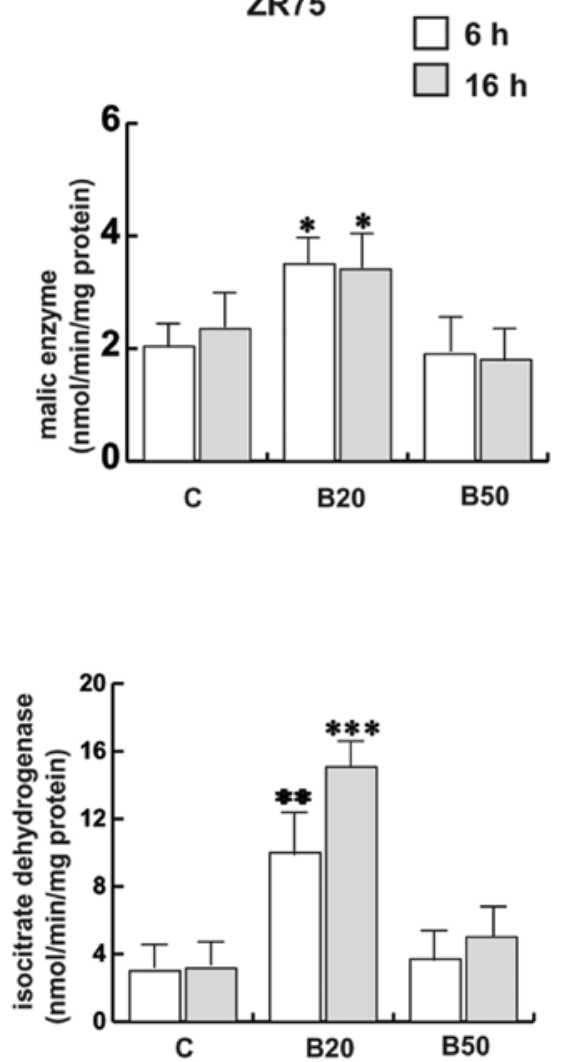

Figure 3. Bg influences the reducing potential in breast cancer cells (A) Malic enzyme assay was performed as reported in Materials and methods and in both cell lines in the absence (C) or with increasing Bg concentrations (B20 $\mu \mathrm{M}$ and B50 $\mu \mathrm{M}$ ) at the indicated times. Columns represent the mean \pm SEM of 20 independent experiments performed in duplicate. ${ }^{*} \mathrm{P}<0.05,{ }^{* *} \mathrm{P}<0.02$ vs. the control (C) at the same time. (B) Isocitrate dehydrogenase activity was performed as reported in Materials and methods. Columns represent the mean $\pm \mathrm{SEM}$ of 20 independent experiments performed in duplicate. ${ }^{*} \mathrm{P}<0.05,{ }^{* *} \mathrm{P}<0.02$. $^{* * *} \mathrm{P}<0.01$ vs. the control (C) at the same time. Bg, bergapten.

appeared to be higher in the ZR75 cells, although not significantly different from that observed in the MCF7 cells Fig. 4D. Following treatment with $\mathrm{Bg}$, our data correlated well with the increased AMPK expression in the MCF7 cells accordingly to the reduced ATP production, while AMPK expression was unchanged in the ZR75 cells, that produced a major amount of ATP.

Bg promotes a lipid-lowering effect in breast cancer cells. The bioenergetic and biosynthetic requirements of cancer cells are balanced by regulating the flux of pathways that metabolize fatty acids other than glucose. Increased fatty acid synthesis has been linked to poor prognosis in breast cancer (22). Bg-treated cells exhibited a reduced triglyceride level compared to the untreated cells, accordingly to the increased lipase activity observed in both cell lines in a time- and concentration-dependent manner (Fig. 5A and B).

Bg-induced metabolic reprogramming produces cell death in breast cancer. As shown in Fig. 6 both MCF7 and ZR75 cells exposed to Bg exhibited a loss of cell survival, although the effect was higher in the MCF7 than that in the ZR75 cells.

\section{Discussion}

It has now been clearly established that cancer cells depend on an altered cellular metabolism (23). Cancers are extremely heterogeneous diseases and each cancer has its individual metabolic features. The literature indicates that the Warburg phenotype is not exclusive and that a decrease in mitochondrial function is not a general feature of cancer cells. In fact, even in glycolytic tumors, OXPHOS is not completely shut down. Herein, we characterized the metabolic profile of MCF7 and ZR75 cells evaluating various features of tumor metabolism. We found that $\mathrm{Bg}$, a natural product, alters both glucose and lipid metabolism in a way and extent that cannot be counterbalanced by breast cancer cells as they die.

Energy metabolism represents an additional hallmark of cancer cells and a new promising target for its treatment. Multiple molecular mechanisms converge to alter core cellular metabolism. Malignant cells have the ability to adopt an altered metabolic profile that fulfills biosynthetic and bioenergetic requirements for rapid and uncontrolled growth. Thus, its disruption may then resolve the malignant process, independently of its origin.

In the present study, detailed attention was given to evaluate the effect of Bg on breast cancer metabolism. Glycolysis is the metabolic program of choice for cells that are actively engaged in cell growth and mitogenesis. Because of this fact, we first analyzed the glucose content and utilization in both MCF7 and ZR75 cells. The basal glucose content was higher in MCF7 than in ZR75 cells and it increased upon $\mathrm{Bg}$ treatment in both cell lines, while the PFK1 expression and the lactate production rates were strongly decreased. 
A

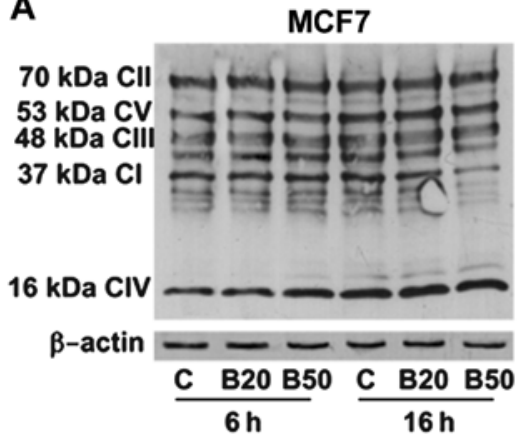

B

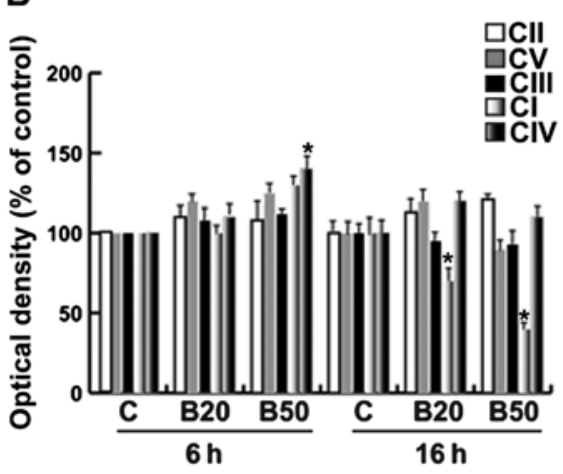

C

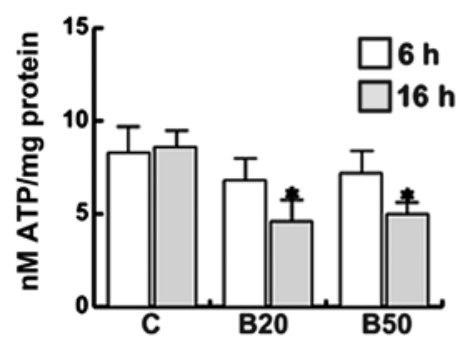

D

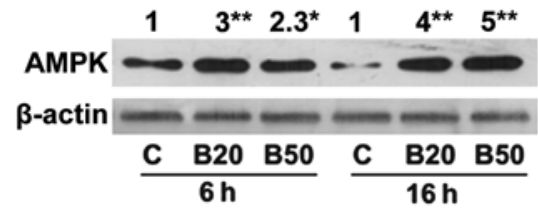

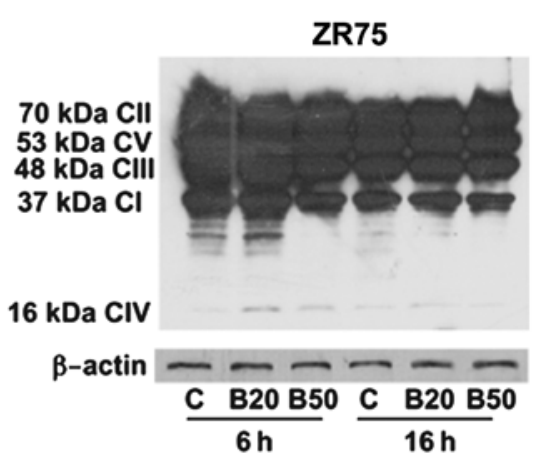
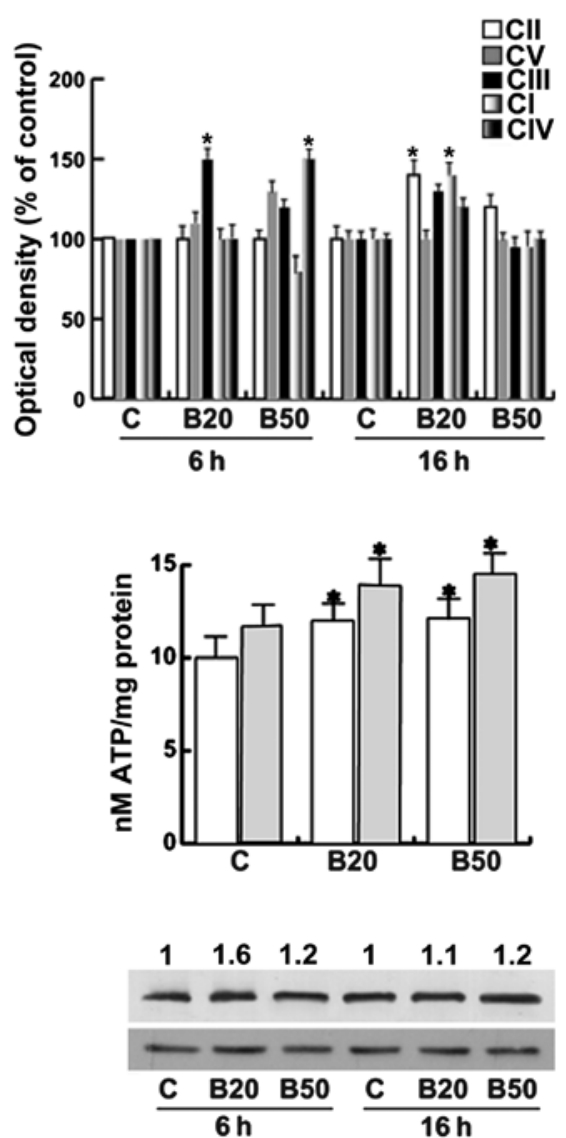

Figure 4. Bg alters the bioenergetics in breast cancer cells. (A) Western blot assay of OXPHOS in both cell lines in the absence (C) or with increasing Bg concentrations (B20 $\mu \mathrm{M}$ and B50 $\mu \mathrm{M})$ at 6 and $16 \mathrm{~h}$. The autoradiographs show the results of one representative experiment repeated at least 3 times. $\beta$-actin was used as a control. (B) Quantitative representation after densitometry of the OXPHOS components. The columns are the mean of three independent experiments in which band intensities were evaluated in terms of optical density arbitrary units and expressed as a percentage with respect to the respective time controls, which were assumed to be $100 \%$. ${ }^{*} \mathrm{P}=0.05,{ }^{*} \mathrm{P}<0.02$. CIV, CI, CIII, CV, CII indicate OXPHOS components. (C) ATP content was assessed as reported in Materials and methods. Columns represent the mean \pm SEM of 20 independent experiments performed in duplicate. $\mathrm{P}<0.05$ vs. the control $(\mathrm{C})$ at the same time; 6 and 16 h.. (D) Western blot assay of AMPK in both cell lines in the absence (C) or with increasing Bg concentrations (B20 $\mu \mathrm{M}$ and B50 $\mu \mathrm{M})$ at the indicated times. $\beta$-actin was used as a control. The autoradiographs show the results of one representative experiment, and the numbers on the top of the blot are mean of 3 independent experiments in which band intensities were evaluated in terms of optical density arbitrary units and expressed as the fold over each control (C), which was assumed to be $1 .{ }^{*} \mathrm{P}<0.02,{ }^{* *} \mathrm{P}<0.01$ vs. the control (C) at the same time. Bg, bergapten.

Elevated production of lactate has been previously correlated with the activation of PFK1, the major regulatory glycolytic enzyme (24). It has been proposed that inhibition of LDH and PFK1 may represent alternative strategies toward the development of anti-glycolytic-based therapies for cancer and in this scenario, the data obtained upon $\mathrm{Bg}$ treatment was well-fit. The LDH activity may be reduced since PFK1 was in turn lowered, however it was not a rate-limiting step in our breast tumor cell lines. It is known that inhibition of PFK-1 results in the accumulation of fructose-6-phosphate, which is then isomerized to glucose-6-phosphate and in turn is diverted into PPP, an important pathway of glucose catabolism representing another feature of the cancer metabolic phenotype. PPP is the primary cellular source of NADPH, the principal intracellular reductant and crucial to fatty acid and amino acid biosynthesis. It has been reported that the enhanced activation of PPP has a number of pro-oncogenic effects (1) and it is known to be hyperactive in cancer $(25,26)$. In this respect, our data showed that $\mathrm{Bg}$ was able to affect the PPP pathway reducing G6PDH activity. The Bg-induced lowering of $\mathrm{LDH}$ and G6PDH 
A
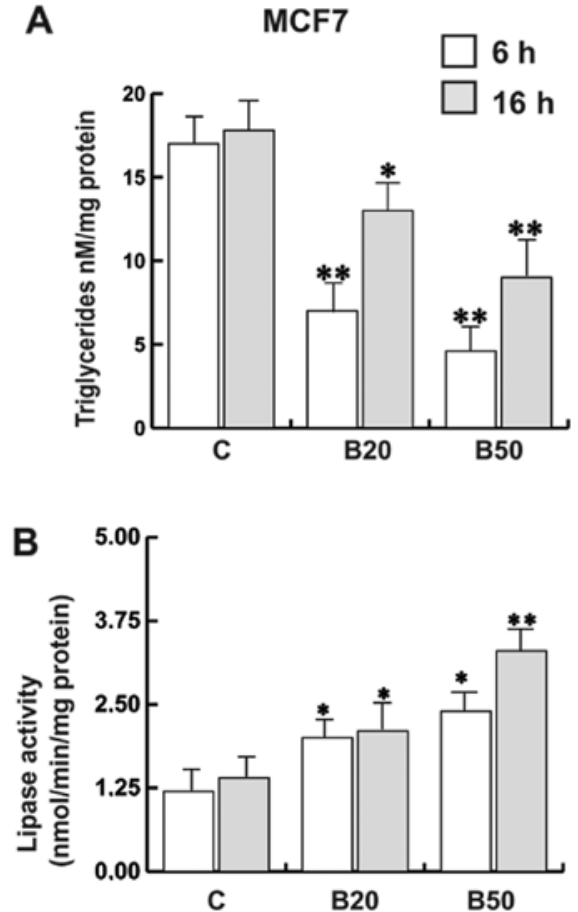
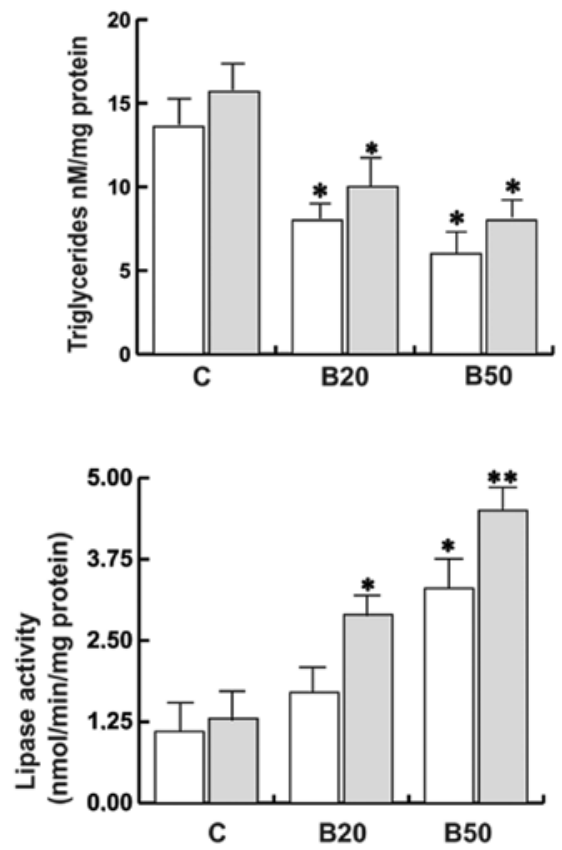

Figure 5. Bg has a lipid-lowering effect in breast cancer cells. (A) Triglyceride assay was performed as reported in Materials and methods and in both cell lines in the absence $(\mathrm{C})$ or with increasing Bg concentrations (B20 $\mu \mathrm{M}$ and B50 $\mu \mathrm{M})$ at the indicated times. Columns represent the mean $\pm \mathrm{SEM}$ of 20 independent experiments performed in duplicate. ${ }^{*} \mathrm{P}<0.05,{ }^{* *} \mathrm{P}<0.01$ vs. the control $(\mathrm{C})$ at the same time. (B) Lipase activity was performed as reported in Materials and methods. Columns represent the mean $\pm \mathrm{SEM}$ of 20 independent experiments performed in duplicate. ${ }^{*} \mathrm{P}<0.05,{ }^{* *} \mathrm{P}<0.01 \mathrm{vs}$. the control $(\mathrm{C})$ at the same time; 6 and $16 \mathrm{~h}$. $\mathrm{Bg}$, bergapten.
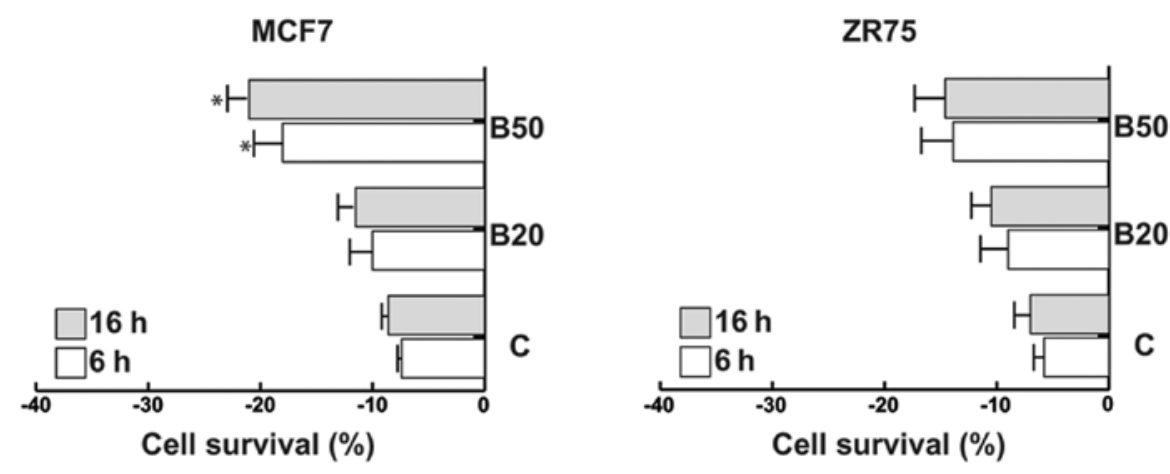

Figure 6. Bg induces cell death in breast cancer cells. (A) Increasing Bg concentrations (B20 $\mu \mathrm{M}$ and B50 $\mu \mathrm{M}$ ) produce loss of cell survival in the MCF7 and ZR75 cells, as assessed by the Trypan blue assay as described in Materials and methods. Columns are the mean \pm SEM of 20 independent experiments performed in duplicate. ${ }^{*} \mathrm{P}<0.05$ vs. untreated cells; 6 and $16 \mathrm{~h}$. Bg, bergapten.

activities may explain the higher glucose levels upon treatment as were obtained. The basal levels of G6PDH activity were lower at $16 \mathrm{~h}$, which may be due to the longer absence of growth factor in the medium used (27). The different LDH and G6PDH basal activities between MCF7 and ZR75 cells that we found, may suggest that the MCF7 cells have a marked glycolytic phenotype when compared with the ZR75 cells. Moreover, the reduction in these enzymatic activities by $\mathrm{Bg}$ indicates that it is able to derange glucose metabolism in breast cancer cells. It appears that $\mathrm{Bg}$ shares similarities with other classes of molecules found in nature within polyphenols, that are widely known as enzyme inhibitors and particularly of glucose metabolism. The repression of the PI3K/Akt activity as we previously demonstrated (12), also impaired anabolic glucose metabolism supporting our new data. Furthermore, $\mathrm{Bg}$ also antagonized the upregulatory effect of insulin on pGSK3S9 expression, an essential control point in glycogen synthesis. It is clear that the absence of energetic reservoirs does not favors cancer cell survival.

Since oncogenic metabolic programs support bioenergetics, we focused our study on NAPDH production, oxidative mitochondrial activity as well as ATP content. Other than PPP, two enzymes are involved in the production of NADPH, ME and ICDH. Bg was able to affect both ME and ICDH activities, by inducing an increase at $20 \mu \mathrm{M}$, while at a higher dose after $16 \mathrm{~h}$ of incubation the level decreased significantly in the MCF7 cells. A similar pattern of response was obtained in ZR75 cells at $6 \mathrm{~h}$; however, treatment with $\mathrm{Bg}$ at $50 \mu \mathrm{M}$ was 
not able to reduce both enzymatic activities. Of consequence, it appears that $\mathrm{Bg}$ acts on NADPH production without biasing these two metabolic steps. Furthermore, as observed for LDH and G6PDH activities, both basal ME and ICDH activities were higher in the MCF7 cells when compred with the activities in the ZR75 cells.

It is well known that oxidation of NADH mainly occurs via mitochondrial OXPHOS, where electron transport is coupled along 4 enzyme complexes (CI-CIV), and ATP is synthesized at CV (ATP synthase) $(28,29)$. From our data, it is evident that treatment with $\mathrm{Bg}$ in MCF7 cells tended to reduce the expression of $\mathrm{CI}$, and then the entrance of the electrons in the mitochondria, although levels of the other downstream components were maintained. This would translate into an internal imbalance that is unable to supply energy to the cells, accordingly to the minor amount of ATP produced. In contrast, in ZR75 cells, the amount of OXPHOS in untreated and Bg-treated cells was higher compared to that observed in the MCF7 cells. This finding supports the increased ATP production in ZR75 cells where a reduction in complex IV of the respiratory chain was noted. Deregulation of these processes is a major pathological consequence of cancer progression.

AMPK is a highly conserved sensor of increased levels of AMP and ADP originating from ATP depletion $(30,31)$. It appears to be involved in cancer because of its ability to act as a tumor-suppressor. From our data, upon Bg treatment, the AMPK content increased in MCF7 cells acting as an energy sensor allowing the cells to meet the reduced energy supply, while in ZR75 cells its expression did not change, in agreement with the higher ATP levels obtained in these cells. Summarizing the role of $\mathrm{Bg}$ on glucose utilization and bioenergetics: in MCF7 cells, upon Bg treatment, glycolysis and PPP rates were significantly reduced, OXPHOS components were scarcely impaired and the ATP production was reduced. In ZR75 cells, the psoralen induced similar effects on glycolysis, while an increased OXPHOS as well as ATP content were observed. These data suggest that the two cell lines exhibit different bioenergetic phenotypes. MCF7 cells express prevalently a glycolytic phenotype only partially oxidative, while ZR75 cells mainly express an oxidative phenotype. This may explain why ZR75 cells are more resistant to B-induced cell death vs. MCF7 cells, as we previously reported (12). This assumption is supported by a recent finding demonstrating how ZR75 cells selectively use genes for energy, sugar metabolism and other pathways differently from MCF7 cells, imparting more aggressiveness to ZR75 cells (24).

The bioenergetic and biosynthetic requirements of cancer cells are balanced by regulating the flux of pathways that metabolize fatty acids other than glucose. Proliferating cells can synthesize fatty acids and cholesterol de novo from glucose and increased fatty acid synthesis has been linked to poor prognosis in breast cancer (32). The dependence of tumor cells on deregulated lipid metabolism suggests that pathways involved in this process are additional attractive targets for cancer treatment. In our study, Bg greatly impacted this feature of cancer cell metabolism since it induced a general lipid-lowering effect. Triglyceride levels decreased concomitantly with an increase in lipase activity. All together, the metabolic reprogramming by $\mathrm{Bg}$ was acutely efficacious to reduce cancer cell survival according to previously reported data.

We report, for the first time, that $\mathrm{Bg}$ treatment interferes with breast cancer cell metabolism disrupting different features of this process. The outcomes emerged from an extensive characterization of the different biochemical pathways involved in cell metabolism. On the basis of these and previous data, the activity of $\mathrm{Bg}$ against breast cancer cells is therefore orchestrated by several components rather than being the result of the effect on a single molecular target and/or signaling pathway.

Future clinical data describing the metabolic profiles of human tumors will be required to determine which metabolic alterations are most prevalent in specific tumor types. In conclusion, bergapten, on the basis of its metabolic targeting, can be used in combination with other forms of targeted chemotherapy to improve cancer treatment outcomes.

\section{Acknowledgements}

The present study was supported by MIUR Ex 60\%-2014 and Associazione Italiana Ricerca sul Cancro (AIRC) (grant no. IG15738). Our special thanks to Dr Vincenzo Cunsolo (Biogemina Italia Srl, Catania, Italy); and Serena Gervasi and Maria Clelia Gervasi for the English language revision of the manuscript.

\section{References}

1. Barger JF and Plas DR: Balancing biosynthesis and bioenergetics: Metabolic programs in oncogenesis. Endocr Relat Cancer 17: R287-R304, 2010.

2. Smolková K, Plecitá-Hlavatá L, Bellance N, Benard G, Rossignol R and Ježek P: Waves of gene regulation suppress and then restore oxidative phosphorylation in cancer cells. Int J Biochem Cell Biol 43: 950-968, 2011.

3. Phan LM, Yeung S-C and Lee M-H: Cancer metabolic reprogramming: importance, main features, and potentials for precise targeted anti-cancer therapies. Cancer Biol Med 11: 1-19, 2014.

4. Vamecq J, Colet JM, Vanden Eynde JJ, Briand G, Porchet N and Rocchi S: PPARs: Interference with Warburg' effect and clinical anticancer trials. PPAR Res 2012: 304760, 2012.

5. Nabekura T: Overcoming multidrug resistance in human cancer cells by natural compounds. Toxins (Basel) 2: 1207-1224, 2010.

6. da Rocha AB, Lopes RM and Schwartsmann G: Natural products in anticancer therapy. Curr Opin Pharmacol 1: 364-369, 2001.

7. Rowinsky EK: Paclitaxel pharmacology and other tumor types. Semin Oncol 2: S19-1-S19-12, 1997.

8. Jordan MA, Toso RJ, Thrower D and Wilson L: Mechanism of mitotic block and inhibition of cell proliferation by Taxol at low concentrations. Proc Natl Acad Sci USA 90: 9552-9556, 1993.

9. Moos PJ and Fitzpatrick FA: Taxanes propagate apoptosis via two cell populations with distinctive cytological and molecular traits. Cell Growth Differ 9: 687-697, 1998.

10. Huang Y, Johnson KR, Norris JS and Fan W: Nuclear factor-kappaB/IkappaB signaling pathway may contribute to the mediation of paclitaxel-induced apoptosis in solid tumor cells Cancer Res 60: 4426-4432, 2000.

11. McDaid HM and Horwitz SB: Selective potentiation of paclitaxel (Taxol)-induced cell death by mitogen-activated protein kinase kinase inhibition in human cancer cell lines. Mol Pharmacol 60: 290-301, 2001.

12. Panno ML, Giordano F, Mastroianni F, Palma MG, Bartella V, Carpino A, Aquila S and Andò S: Breast cancer cell survival signal is affected by bergapten combined with an ultraviolet irradiation. FEBS Lett 584: 2321-2326, 2010.

13. Panno ML, Giordano F, Palma MG, Bartella V, Rago V, Maggiolini M, Sisci D, Lanzino M, De Amicis F and Andò S: Evidence that bergapten, independently of its photoactivation, enhances p53 gene expression and induces apoptosis in human breast cancer cells. Curr Cancer Drug Targets 9: 469-481, 2009. 
14. Guido C, Panza S, Santoro M, Avena P, Panno ML, Perrotta I, Giordano F, Casaburi I, Catalano S, De Amicis F, et al: Estrogen receptor beta $(\mathrm{ER} \beta)$ produces autophagy and necroptosis in human seminoma cell line through the binding of the Sp1 on the phosphatase and tensin homolog deleted from chromosome 10 (PTEN) promoter gene. Cell Cycle 11: 2911-2921, 2012.

15. Saifer A and Gerstenfeld S: The photometric microdetermination of blood glucose with glucose oxidase. J Lab Clin Med 51: 448-460, 1958.

16. Guido C, Perrotta I, Panza S, Middea E, Avena P, Santoro M, Marsico S, Imbrogno P, Andò S and Aquila S: Human sperm physiology: Estrogen receptor alpha $(\mathrm{ER} \alpha)$ and estrogen receptor beta $(E R \beta)$ influence sperm metabolism and may be involved in the pathophysiology of varicocele-associated male infertility. J Cell Physiol 226: 3403-3412, 2011.

17. Panteghini M, Bonora R and Pagani F: Measurement of pancreatic lipase activity in serum by a kinetic colorimetric assay using a new chromogenic substrate. Ann Clin Biochem 38: 365-370, 2001.

18. Aquila S, Bonofiglio D, Gentile M, Middea E, Gabriele S, Belmonte M, Catalano S, Pellegrino M and Andò S: Peroxisome proliferator-activated receptor (PPAR)gamma is expressed by human spermatozoa: Its potential role on the sperm physiology. $\mathrm{J}$ Cell Physiol 209: 977-986, 2006.

19. Pingitore A, Cione E, Senatore V and Genchi G: Adrenal glands and testes as steroidogenic tissue are affected by retinoylation reaction. J Bioenerg Biomembr 41: 215-221, 2009.

20. Gnoni GV and Paglialonga G: Resveratrol inhibits fatty acid and triacylglycerol synthesis in rat hepatocytes. Eur J Clin Invest 39: 211-218, 2009.

21. Luo J: Glycogen synthase kinase 3beta (GSK3beta) in tumorigenesis and cancer chemotherapy. Cancer Lett 273: 194-200, 2009.

22. Zhang F and Du G: Dysregulated lipid metabolism in cancer. World J Biol Chem 3: 167-174, 2012.
23. Phan LM, Yeung SC and Lee MH: Cancer metabolic reprogramming: Importance, main features, and potentials for precise targeted anti-cancer therapies. Cancer Biol Med 11: 1-19, 2014.

24. Mandal S and Davie JR: An integrated analysis of genes and pathways exhibiting metabolic differences between estrogen receptor positive breast cancer cells. BMC Cancer 7: 181, 2007.

25. Richardson AD, Yang C, Osterman A and Smith JW: Central carbon metabolism in the progression of mammary carcinoma. Breast Cancer Res Treat 110: 297-307, 2008.

26. Meadows AL, Kong B, Berdichevsky M, Roy S, Rosiva R, Blanch HW and Clark DS: Metabolic and morphological differences between rapidly proliferating cancerous and normal breast epithelial cells. Biotechnol Prog 24: 334-341, 2008.

27. Tian WN, Braunstein LD, Pang J, Stuhlmeier KM, Xi QC, Tian X and Stanton RC: Importance of glucose-6-phosphate dehydrogenase activity for cell growth. J Biol Chem 273: 10609-10617, 1998.

28. Sun X, Wang JF, Tseng M and Young LT: Downregulation in components of the mitochondrial electron transport chain in the postmortem frontal cortex of subjects with bipolar disorder. J Psychiatry Neurosci 31: 189-196, 2006.

29. Reinecke F, Smeitink JA and van der Westhuizen FH: OXPHOS gene expression and control in mitochondrial disorders. Biochim Biophys Acta 1792: 1113-1121, 2009.

30. Hardie DG: AMP-activated protein kinase: An energy sensor that regulates all aspects of cell function. Genes Dev 25: 1895-1908, 2011.

31. Mihaylova MM and Shaw RJ: The AMPK signalling pathway coordinates cell growth, autophagy and metabolism. Nat Cell Biol 13: 1016-1023, 2011.

32. Shurbaji MS, Kalbfleisch JH and Thurmond TS: Immunohistochemical detection of a fatty acid synthase (OA-519) as a predictor of progression of prostate cancer. Hum Pathol 27: 917-921, 1996. 